\title{
T-junctions, apparent depth, and perceived lightness contrast
}

\author{
FREDERICK BONATO \\ Saint Peter's College, Jersey City, New Jersey \\ JOSEPH CATALIOTTI \\ Ramapo College, Mahwah, New Jersey \\ and \\ MELISSA MANENTE and KAREN DELNERO \\ Saint Peter's College, Jersey City, New Jersey
}

\begin{abstract}
Observers performed lightness matches for physically equivalent gray targets of a simultaneous lightness contrast display and displays in which both targets were on the same background. Targets either shared a common line-texture pattern with their respective backgrounds or did not. Results indicate that when targets share a line-texture pattern with their respective backgrounds, a contrast effect is obtained. However, when the target's pattern is different than the background's pattern, perceived contrast is significantly reduced and the target appears as a separate $3-\mathrm{D}$ entity. This result applies to both vertically and horizontally oriented displays, to targets that are increments or decrements, and to line-texture patterns that are black or white. Line patterns that are shared by targets and backgrounds result in T-junctions that provide occlusion information. We conclude that targets and backgrounds perceived to be on separate planes because of T-junctions are less likely to be perceptually grouped together and that their luminance values are less likely to be compared with one another.
\end{abstract}

A considerable amount of debate has taken place concerning the cause of simultaneous lightness contrast (SLC). In this well-known illusion, displayed in Figure 1, the two gray targets appear to be different in lightness even though they have the same reflectance. SLC is more than just a curiosity; any complete theory of lightness perception must be able to explain it. SLC represents a systematic error committed by the visual system, and understanding it can offer clues about how lightness values are determined. Historically, explanations of SLC have fallen into two general categories.

Some explanations (see, e.g., Diamond, 1960; Nabet \& Pinter, 1991; Ratliff, 1965; Shapley, Caelli, Grossberg, Morgan, \& Rentschler,1990) of SLC are based on lateral inhibition. Studying the horseshoe crab, limulus, Hartline, Wagner, and Ratliff (1956) discovered that stimulated retinal receptors inhibit the firing rates of neighboring cells. Applied to SLC, the sites on the retina or lateral geniculate nucleus corresponding to the two gray targets are equally stimulated, but unequally inhibited. Sites corresponding to the target surrounded by white receive a high level of inhibition, whereas the target surrounded by

This research was supported by National Science Foundation Grants BCS-0002620 and BCS-0196013. The authors thank Bart Anderson and an anonymous reviewer for their helpful comments regarding this manuscript. Correspondence concerning this article should be addressed to F. Bonato, Department of Psychology, Saint Peter's College, 2641 Kennedy Boulevard, Jersey City, NJ 07306 (e-mail: bonato_f @ spc.edu). black receives less inhibition. The lateral inhibition explanation treats SLC as a perceptual by-product of the differential firing rates of cells that correspond to the two target images. Some lightness theorists (e.g., Cornsweet, 1970; Jameson \& Hurvich, 1961,1964) have favored the lateral inhibition explanation of SLC and have used lateral inhibition as a foundation for their theories of lightness perception.

One problem for a lateral inhibition account of SLC is that the amount of inhibition exerted on neighboring cells should theoretically weaken with distance. Thus, retinal lateral inhibition should serve as an edge enhancement mechanism because the firing rate profile does not correspond to the luminance profile of the scene being viewed. Although Mach bands are often used as examples to illustrate the "scalloped" firing rate pattern produced by lateral inhibition, no such perceptual correlate seems noticeable in SLC. The firing rate profile produced by lateral inhibition should yield targets whose edges appear more different than their respective backgrounds in terms of lightness, yet the targets appear to be homogeneous. Some have accounted for the homogeneous appearance of SLC targets by postulating the existence of a "filling-in" mechanism.

Also, lateral inhibition in its purest form is structure blind, in that it would predict the same results regardless of whether the edges in a given display are caused by changes in reflectance or changes in illumination (shadows). Gilchrist (1988) has shown that the targets of an SLC 


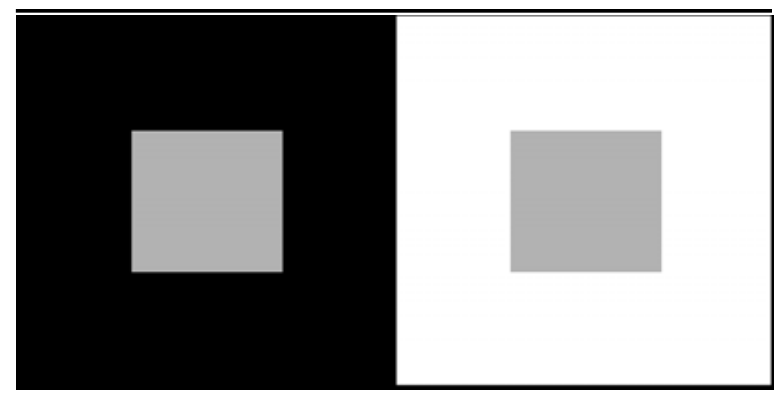

Figure 1. Simultaneous lightness contrast. The two gray squares have equal reflectance values and luminance values, but the square surrounded by white appears darker than the square surrounded by black.

pattern are matched very much differently depending on whether the edge that separates the light and dark backgrounds is perceived as a reflectance edge or as an illumination edge. When the dark background is perceived as the shadowed half of a surface having only one reflectance, the target in the shadowed region appears to be much lighter than a target of equal luminance on a dark background that is perceived as having a lower reflectance.

Other explanations of SLC have emphasized perceptual grouping and have stressed the importance of higher level processing that presumably occurs beyond the retinal level in the visual pathway. Some of these explanations owe much to the laws of perceptual organization first outlined by the early Gestaltists. It could be argued that each gray target of an SLC display is grouped with its respective background because of proximity, surroundedness, and coplanarity. Early work by Benary (1924) and Wolff (1933) tried to show the importance of perceptual grouping by making regions appear different in lightness, despite equal retinal stimulation and inhibition.

Recently, other investigators have stressed the importance of perceptual organization in relation to contrast effects. Gilchrist and colleagues (Gilchrist et al., 1999) have recently proposed a theory of lightness perception in which perceptual grouping plays a pivotal role; their theory addresses contrast effects, including SLC. Anderson (1997) has proposed a theory of contrast in which targets in various types of displays undergo scission into multiple layers. The luminance of a given target is the combination of a reflectance layer and a transparency or illumination layer. The results of the present study and how they relate to these two theories will be discussed later in more detail.

Agostini and Proffitt (1993) showed that a gray region moving in concert with a group of white regions appears darker than a gray region of identical luminance that moves in concert with a group of black regions. Both gray regions were on the same blue background and as a result are equally influenced by retinal excitation and inhibition. By using various grouping principles (similarity, proximity, good continuation) Economou, Annan, and Gilchrist (1998) have demonstrated a version of SLC in which the gray target on black appears darker, not lighter, than the gray target surrounded by white. A lat- eral inhibition theory of SLC would predict a result that goes in the opposite direction.

Adelson (1993) has demonstrated several compelling illusions that can perhaps best be explained in terms of grouping processes that occur beyond the level of the retina. Laurinen, Olzak, and Peromaa (1997) showed that the magnitude of SLC can be weakened by superimposing modulation patterns on the targets that are different than the patterns superimposed onto the targets' backgrounds. They claimed that differential target/background modulation causes the targets to be seen as separate 3-D entities that contrast less with their respective backgrounds, an idea that goes back as far as Wolff (1933), who suspended SLC targets in front of their respective backgrounds and obtained a reduction in perceived lightness contrast.

One of the most compelling examples of a contrast effect that cannot be easily explained by retinal lateral inhibition is White's effect (White, 1981), which is shown in Figure 2. In this illusion two gray targets of equal luminance appear to be different in lightness, but in the direction opposite to that which would be predicted by lateral inhibition. Specifically, gray bars that are surrounded mostly by black appear darker than gray bars that are surrounded mostly by white. White's effect is especially relevant to the present study because it is thoughtby some (e.g., Anderson, 1997; Gilchrist et. al., 1999) that T-junctions contribute to the illusion.

The present study was conducted to investigate how superimposed line-texture patterns that produce T-junctions can affect lightness contrast. T-junctions often provide information about occlusion and thus a separation in depth. The present study differs from the work of Wolff (1933) and Laurinen et al. (1997) in the following ways:

1. Texture patterns that produce T-junctions were the focus of the present study.

2. Many investigators have studied perceived lightness contrast by using several versions of the classic SLC display. Here, we present experiments in which targets were presented on the same background. This manipulation allows one to isolate the effects of modulation patterns on the lightness of the targets.

3. When a version of a classic SLC display is used, it is usually unclear which target is being affected - the target surrounded by black (increment) or the target surrounded

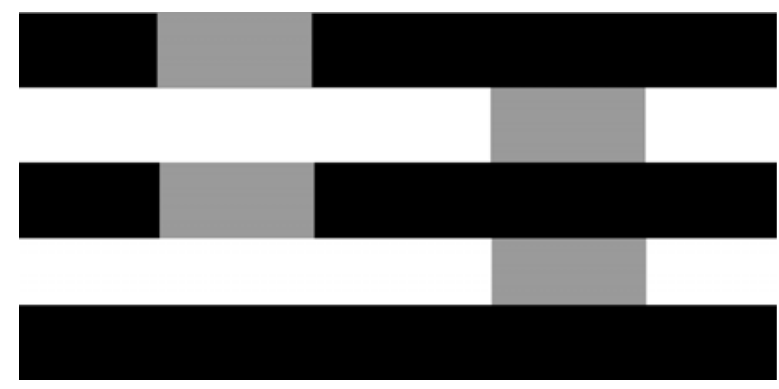

Figure 2. White's effect. The gray bars on the left and right have equal reflectance values and luminance values, but the bars on the left appear lighter than the bars on the right. 
by white (decrement). In the present study, increments and decrements were tested separately in order to determine whether line-texture patterns affect increments, decrements, or both.

4. Laurinen et al. (1997) claimed that differential target/ background modulation caused their targets to be seen as separate 3-D entities. We recorded observers' depth impressions of the displays in the present study in order to obtain a more complete account of their perceptual experiences, especially those pertaining to depth.

5. The effects of black and white line-texture patterns were tested separately.

In the first experiment, we tested the effect of T-junction producing line patterns on SLC. Unlike Laurinen et al. (1997), who used modulation patterns that gradually changed (e.g., sinusoidal patterns), the stimuli in Experiment 1 were superimposed with simple line patterns.

\section{EXPERIMENT 1 Simultaneous Lightness Contrast}

\section{Method}

Stimulus displays. The two stimulus displays that were presented are shown in Figure 3. Each display consisted of a $6.5 \times 13 \mathrm{~cm}\left(3.0^{\circ} \times\right.$ $6.0^{\circ}$ ) SLC display centered on a white background (reflectance $=90 \%$, $\left.52 \mathrm{~cd} / \mathrm{m}^{2}\right)$ that was $22 \mathrm{~cm}$ wide and $28 \mathrm{~cm}$ high $\left(10.0^{\circ} \times 12.6^{\circ}\right)$. Each gray $\left(\right.$ reflectance $\left.=43 \%, 24 \mathrm{~cd} / \mathrm{m}^{2}\right)$ target was a $2.5-\mathrm{cm}$ square $\left(1.15^{\circ}\right)$. One target was centered on the white (reflectance $=90 \%$, $52 \mathrm{~cd} / \mathrm{m}^{2}$ ) half of the display, and the other target was centered on the gray (reflectance $=16 \%, 10 \mathrm{~cd} / \mathrm{m}^{2}$ ) half of the display. Targets and backgrounds were superimposed with black line (reflectance $=$ $3 \%, 3 \mathrm{~cd} / \mathrm{m}^{2}$ ) grids consisting of lines $0.4 \mathrm{~mm}$ thick spaced $3 \mathrm{~mm}$ apart. On the SLC display referred to as texture invariant, the targets and backgrounds were superimposed with the same grid pattern. On the SLC display referred to as texture variant, the grid pattern superimposed onto the background regions was rotated $45^{\circ}$.

Laboratory arrangem ents. A standing observer viewed the stimulus display binocularly from a distance of $1.25 \mathrm{~m}$. The stimulus was positioned at a height of $1.65 \mathrm{~m}$ and was attached to a white $\left(40 \mathrm{~cd} / \mathrm{m}^{2}\right)$ background that was perpendicular to the observer's line of sight. The display was illuminated by ambient light from ceilingmounted fluorescent bulbs. For the purpose of allowing lightness matches, a chart of 16 matte finish achromatic Munsell chips was placed on a $91-\mathrm{cm}$-high table, $40 \mathrm{~cm}$ in front of the observer. The $1 \times 3 \mathrm{~cm}$ chips ranged from black (reflectance $=3 \%$ ) to white (reflectance $=90 \%$ ) and were mounted incrementally on an $11 \times 35 \mathrm{~cm}$ white background. The chart was placed on the bottom of a light box and was illuminated from above by a fluorescent bulb. This lighting arrangement resulted in the white chip's having a luminance value of $377 \mathrm{~cd} / \mathrm{m}^{2}$.

Procedure. The observer was instructed to stand in front of the stimulus display at the proper viewing distance, at which time the experimenter asked the observer to pay attention to one of the gray targets. The experimenter then instructed the observer to choose from the Munsell chart the chip that most closely matched the gray shade of the target. The experimenter recorded the observer's lightness match before repeating the same procedure for the other tar-

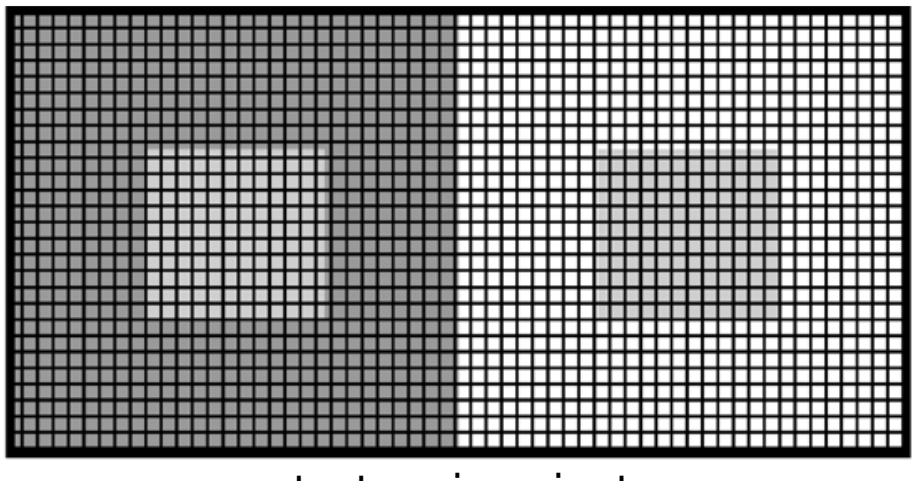

texture-invariant

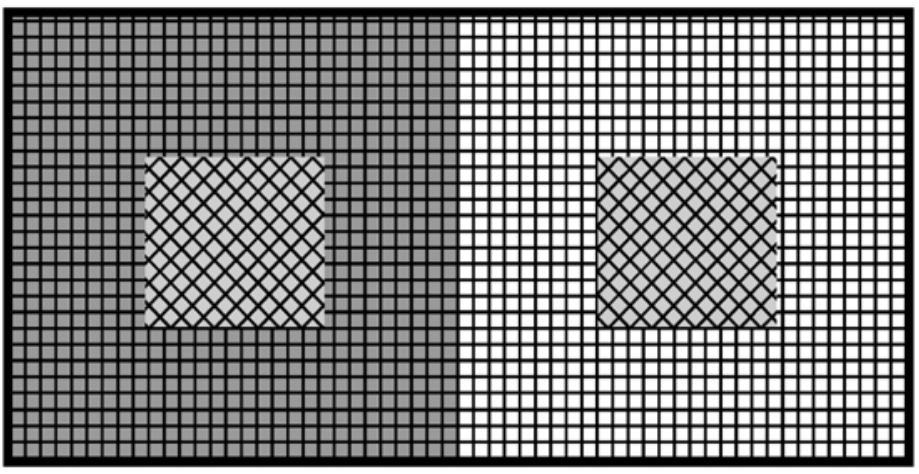

texture-variant

Figure 3. Stimulus displays for Experiment 1. 
get. After the two lightness matches were recorded, the observer was asked to describe any additional visual impressions experienced while he/she was looking at the display, especially those that involved apparent depth relationships between the various elements of the display.

Design. Twenty-four observers made lightness matches for both gray targets in the texture-invariant display, and 24 other observers made lightness matches for the targets on the texture-variant display. The order in which the observers made lightness judgments for the two targets was counterbalanced.

Observers. Forty-eight experimentally naive undergraduate students from Saint Peter's College served as observers.

\section{Results and Discussion}

The mean lightness matches for the target squares are shown in Figure 4. For the texture-invariant display, the mean match for the target on the white background $(M=$ $32 \%, S D=10.4$ ) was significantly lower $[t(23)=5.1, p<$ .0001] than the mean match for the target on the gray $(M=43 \%, S D=11.4)$. This result is not surprising, because it simply demonstrates a typical SLC effect. However, for the texture-variant display, the mean match for the target on white $(M=37 \%, S D=7.4)$ was not significantly darker $[t(23)=1.99, p=.059]$ than the mean match obtained for the target on gray $(M=40 \%, S D=$
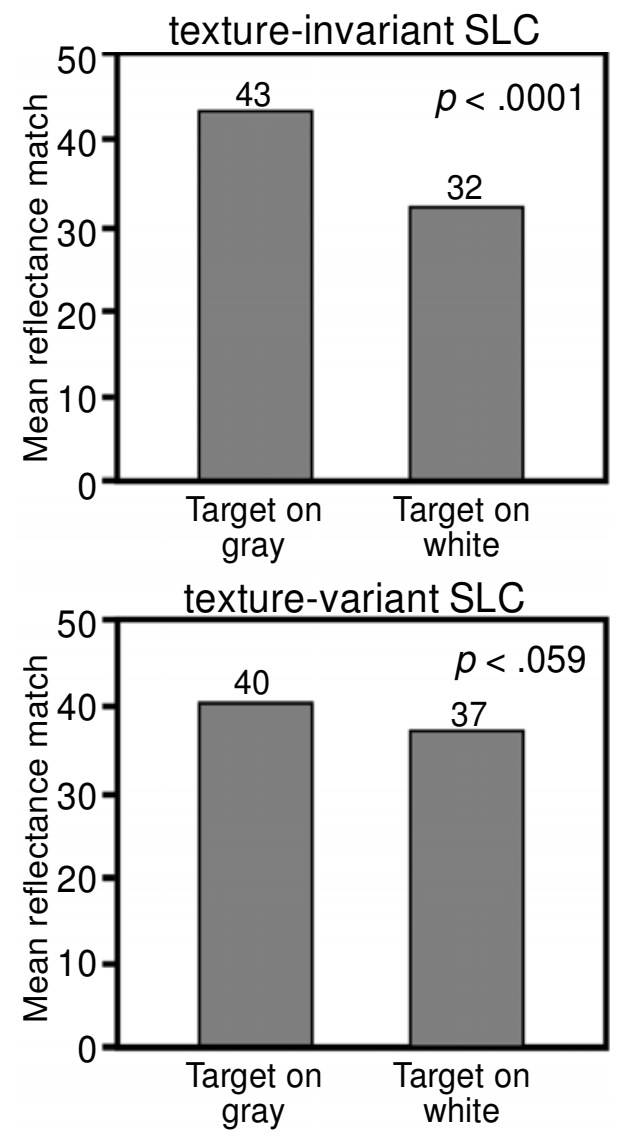

Figure 4. Results of Experiment 1. A significant contrast effect was obtained for the texture-invariant simultaneous lightness contrast (SLC) display but not for the texture-variant SLC display.
8.7). This result strongly suggests that the superimposed lines on the texture-variant display significantly reduced perceived contrast.

Within each experimental condition, there was unanimous agreement among the observers regarding their impressions of target depth. All the observers who served in the texture-invariant condition reported that both targets appeared to be positioned behind the background lines. All the observers who served in the texture-variant condition reported that the targets appeared as separate 3 -D entities in front of the background lines. These results are in agreement with those obtained by Laurinen et al. (1997) and Wolff (1933), who found that when the targets of an SLC display are perceived to be on a plane different from that of the background, the targets appear more alike in lightness. However, whereas those investigators used other means to achieve a depth separation between the targets and their backgrounds, we used line patterns that produced T-junctions.

The results of Experiment 1 cannot be explained by retinal lateral inhibition alone. One has to assume that retinal excitatory and inhibitory processes were the same for the texture-invariant and texture-variant displays. Processing that occurs higher in the visual pathways must play a role.

In the displays used in Experiment 1, targets were presented on different backgrounds, making it unclear how much each target, if any, was affected by retinal processes. Although in the present study, we did not eliminate any retinal inhibitory processes that might have influenced the appearance of the targets, we did equate those influences in Experiment 2. In order to isolate the effects of line-texture patterns, Experiments 2 and 3 were conducted with the use of stimulus displays in which both targets were presented on the same background. This ensured that retinal lateral inhibition would be the same for both targets; the resulting effects would be necessarily caused by the line-texture patterns alone.

\section{EXPERIMENT 2 Same Background, Black Lines}

\section{Method}

Stimulus displays. The two stimulus displays are shown in Figure 5. Each display was $25 \mathrm{~cm}$ wide and $19 \mathrm{~cm}$ high $\left(11.3^{\circ} \times 6.4^{\circ}\right)$ and was superimposed with a pattern of parallel black lines (reflectance $\left.=3 \%, 3 \mathrm{~cd} / \mathrm{m}^{2}\right) 0.4 \mathrm{~mm}$ thick and spaced $3.0 \mathrm{~mm}$ apart. The background for the increment display was gray (reflectance $=$ $16 \%, 10 \mathrm{~cd} / \mathrm{m}^{2}$ ), and the background for the decrement display was white $\left(\right.$ reflectance $\left.=90 \%, 52 \mathrm{~cd} / \mathrm{m}^{2}\right)$. Two $2.5-\mathrm{cm}\left(1.15^{\circ}\right)$ gray (reflectance $\left.=30 \%, 21 \mathrm{~cd} / \mathrm{m}^{2}\right)$ target squares were mounted $5 \mathrm{~cm}\left(2.25^{\circ}\right)$ apart, in the center of each stimulus display. The texture-invariant target was superimposed with the same line pattern that was on the background. The texture-variant target was superimposed with a line pattern that was perpendicular to the background's pattern.

Laboratory arrangements and Procedure. Depending on the condition, the stimulus display was oriented either horizontally or vertically. The procedure was identical to that in Experiment 1 .

Design. A group of 20 observers made lightness matches for the texture-invariant and texture-variant targets on the increment and 


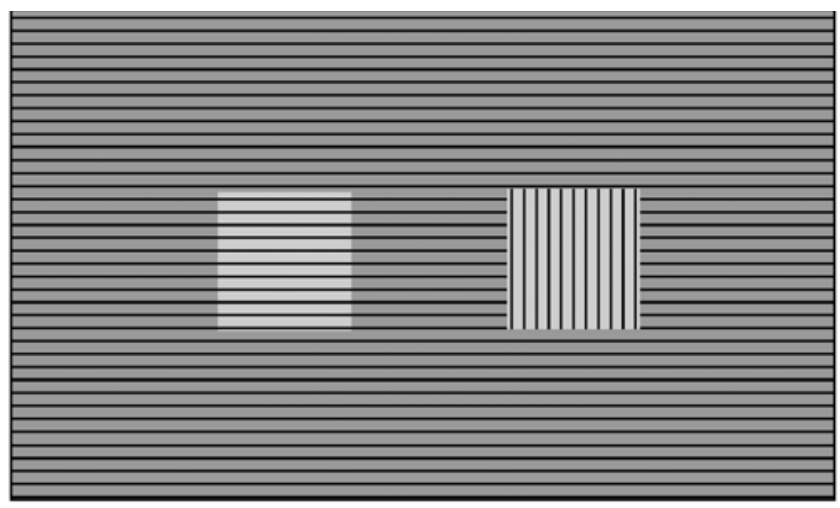

Increment Display

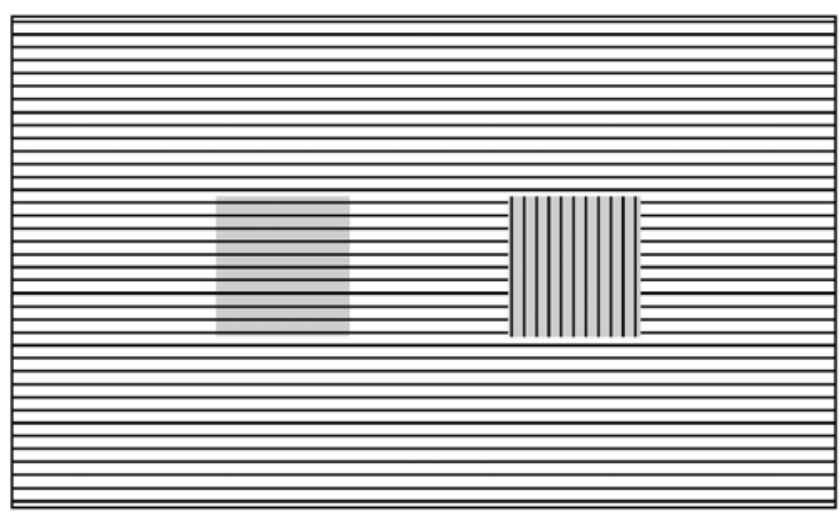

Decrement Display

Figure 5. Stimulus displays for Experiment 2.

decrement displays that were horizontally oriented. A second group of 20 observers made matches for the same displays that were vertically oriented (rotated $90^{\circ}$ ). The order of matches was counterbalanced across conditions.

Observers. Forty experimentally naive undergraduate students from Saint Peter's College served as observers.

\section{Results and Discussion}

The mean lightness matches obtained for the targets are shown in Figure 6. The following matches were obtained for the horizontally oriented displays. In the increment condition, the mean lightness for the texture-invariant target $(M=64 \%, S D=13.4)$ was significantly lighter $[t(19)=$ $3.31, p<.004]$ than the mean match for the texture-variant target $(M=57 \%, S D=13.6)$. In the decrement condition, the mean lightness for the texture-invariant target $(M=$ $45 \%, S D=8.0$ ) was significantly darker $[t(19)=2.48$, $p<.023]$ than the mean match for the texture-variant target $(M=51 \%, S D=7.5)$.

Similar results were obtained for the targets on the vertically oriented displays. In the increment condition, the mean lightness for the texture-invariant target $(M=$ $61.0 \%, S D=7.1)$ was significantly lighter $[t(19)=4.00$, $p<.001]$ than the mean match for the texture-variant target $(M=53 \%, S D=10.8)$. In the decrement condition, the mean lightness for the texture-invariant target $(M=46 \%$, $S D=9.5)$ was significantly darker $[t(19)=2.48, p<$ $.048]$ than the mean match for the texture-variant target $(M=51 \%, S D=11.3)$.

Across conditions, a common result was obtained: All texture-invariant targets contrasted more with their backgrounds than did their texture-variant counterparts. Specifically, the texture-invariant increments appeared lighter than the texture-variant increments, and the textureinvariant decrements appeared darker than the texturevariant decrements.

These results build on Laurinen et al.'s (1997) in two ways: (1) The same pattern of results was obtained for both horizontally and vertically oriented displays, suggesting that the effect is not caused by line orientation per se. One might expect this to be an obvious result, and yet, there are perceptual effects such as the oblique effect and $\mathrm{T}$ illusion that depend on orientation. Each target's line pattern relative to the background's pattern seemed to be the important factor. (2) In Laurinen et al.'s study, it is unclear which target was affected by the modulation patterns. In the present study, targets that have a higher luminance than their background (increments) and targets that have a lower luminance than their back- 

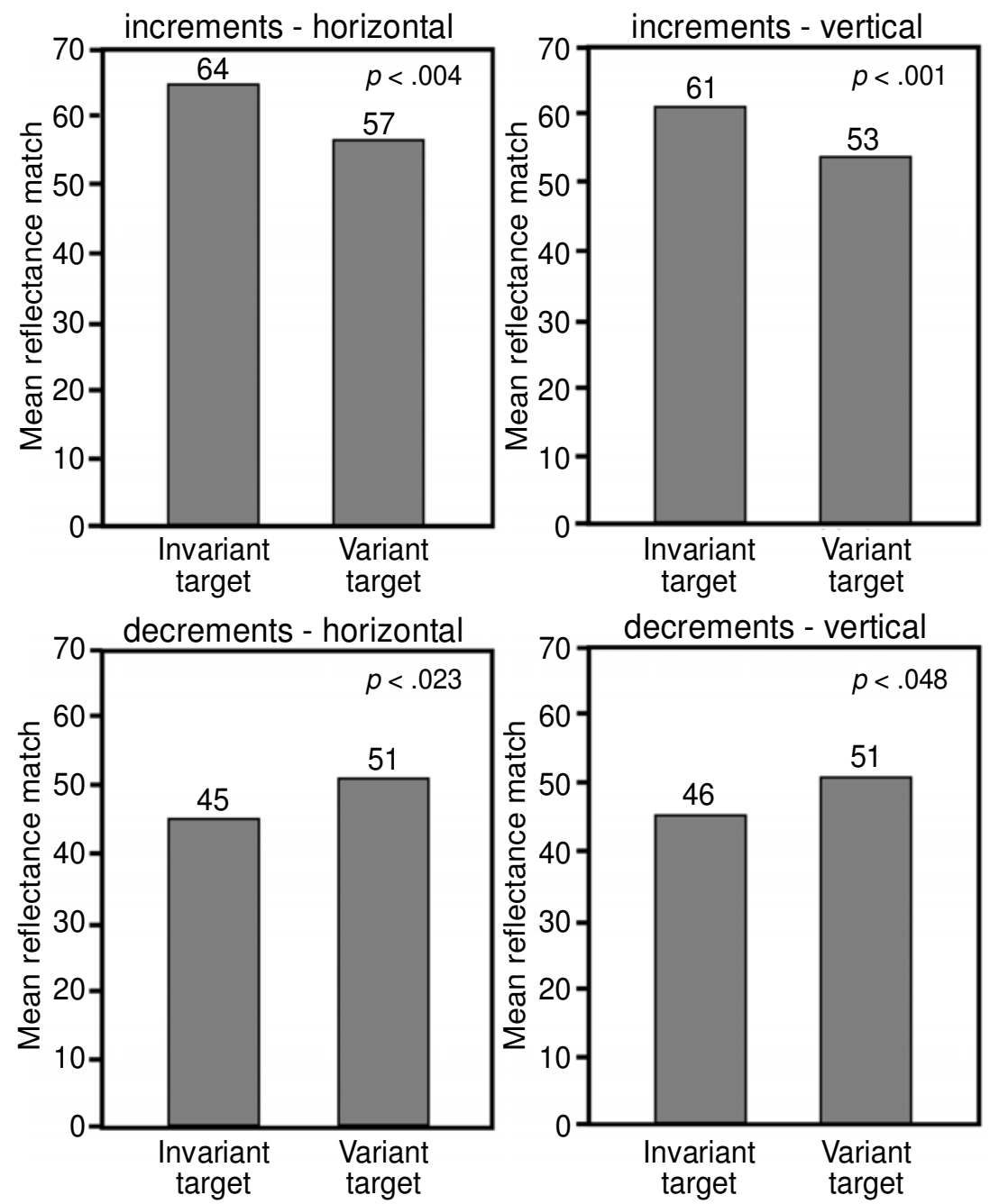

Figure 6. Results of Experiment 2 using black line-texture patterns. For both horizontally and vertically oriented displays, the texture-invariant targets were judged to be more different than their respective backgrounds in terms of lightness.

grounds (decrements) are both affected by line-texture patterns.

As in Experiment 1, the observers in Experiment 2 unanimously agreed that the texture-variant targets appeared to pop out in front of the displays and appeared as 3 -D entities that were not coplanar with their respective backgrounds. However, the observers always reported a dual experience when looking at the displays. Although, in some sense, the texture-variant targets appeared to be located on a plane in front of the background, when asked whether the texture-variant targets were really located on a separate plane, all the observers agreed that they were not.

The display patterns in Experiment 2 were similar to assimilation displays in which homogenous surfaces are superimposed with patterns of thin lines. In these types of assimilation displays, a homogeneous gray background superimposed with thin black lines appears darker than an identical gray background superimposed with white lines. The differences between assimilation displays and our displays are that our displays contained target regions, and the texture-variant target was superimposed with a line pattern that was perpendicular to the pattern on the rest of the display. One could hypothesize that some sort of an assimilation effect was responsible for the results of Experiment 2. For the decrement displays, such a hypothesis might state that the texture-invariant target assimilates to the black lines more than does the texture-variant target. Indeed, one could argue that the line pattern on the texture-variant target is figural, and as a result, it sets up a contrast situation (Coren, 1969). However, such a hypothesis must be rejected, at least for the increment display. If assimilation affected the texture- 
invariant target more on the increment display, the textureinvariant target should have appeared darker, not lighter, than the texture-variant target.

Experiment 3 was conducted with the use of displays superimposed with white lines instead of black lines in order to test the effects, if any, of line color.

\section{EXPERIMENT 3 Same Background, White Lines}

\section{Method}

The method in Experiment 3 was identical to that in Experiment 2, except for the stimulus displays, which were superimposed with white line (reflectance $=90 \%, 52 \mathrm{~cd} / \mathrm{m}^{2}$ ) patterns instead of black ones. In order for the white line pattern to be seen on the decrement display, the white background in Experiment 2 was replaced with light gray $\left(\right.$ reflectance $=59 \%, 41 \mathrm{~cd} / \mathrm{m}^{2}$ ). The target squares on both the increment and decrement displays were gray (reflectance $\left.=30 \%, 21 \mathrm{~cd} / \mathrm{m}^{2}\right)$.

\section{Results and Discussion}

The mean lightness matches obtained for the targets are shown in Figure 7. The following matches were obtained for the horizontally oriented displays. In the increment condition, the mean lightness for the texture-invariant target $(M=51 \%, S D=9.9)$ was significantly lighter $[t(19)=$ $7.59, p<.0001]$ than the mean match for the texturevariant target $(M=40 \%, S D=10.1)$. In the decrement condition, the mean lightness for the texture-invariant target $(M=30 \%, S D=14.9)$ was significantly darker $[t(19)=$ $11.1, p<.0001]$ than the mean match for the texturevariant target $(M=38 \%, S D=11.5)$.

For the targets on the vertically oriented displays, in the increment condition, the mean lightness for the texture-
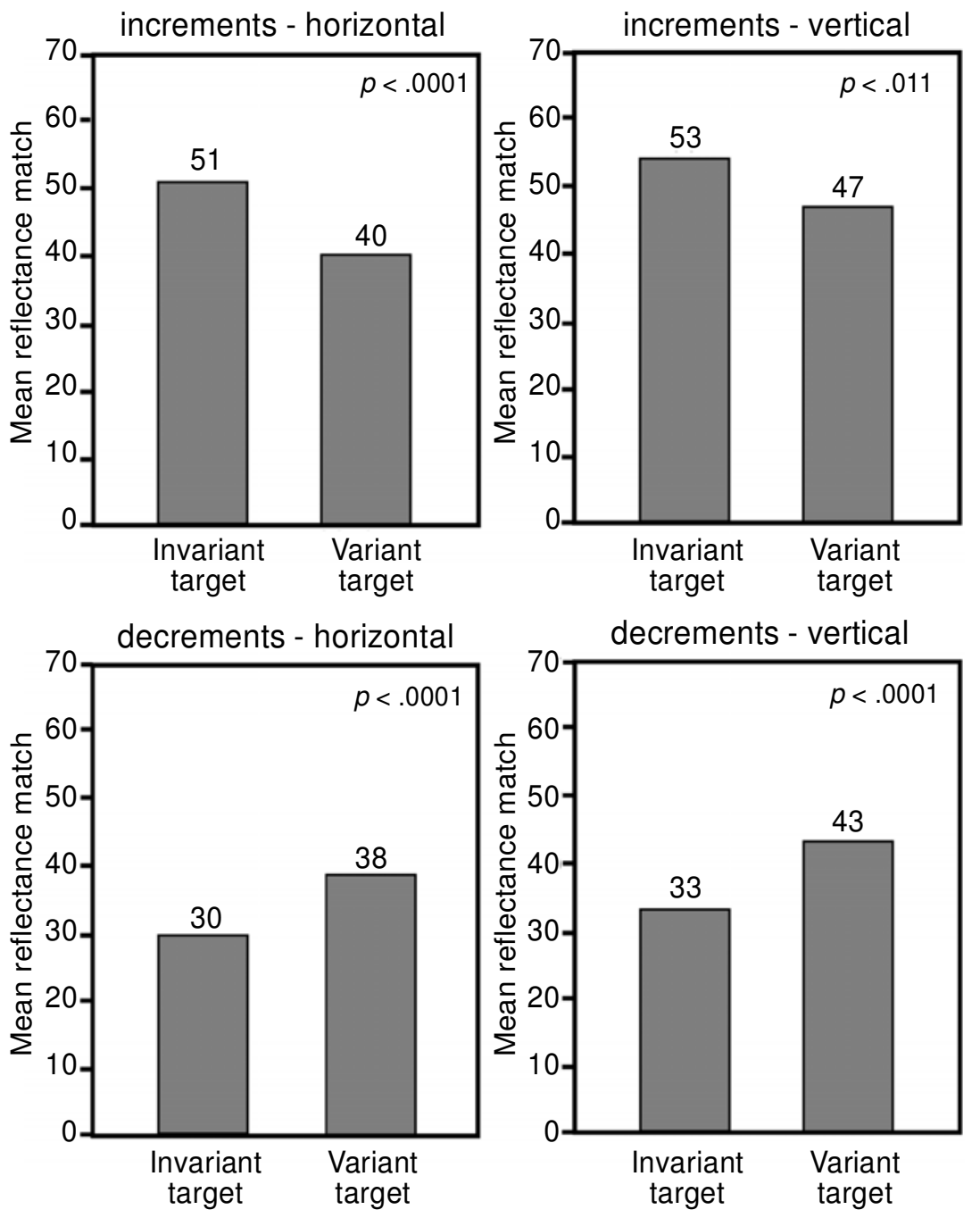

Figure 7. Results of Experiment 3 using white line-texture patterns. As in Experiment 2, for both horizontally and vertically oriented displays, the texture-invariant targets were judged to be more different than their respective backgrounds in terms of lightness. 
invariant target $(M=53 \%, S D=15.0)$ was significantly lighter $[t(19)=2.8, p<.011]$ than the mean match for the texture-variant target $(M=47 \%, S D=11.5)$. In the decrement condition, the mean lightness for the texture-invariant target $(M=33 \%, S D=10.2)$ was significantly darker $[t(19)=4.35, p<.0001]$ than the mean match for the texture-variant target $(M=43 \%, S D=10.4)$.

These results clearly indicate that with white or black line patterns, the general pattern of results is the same: Texture-invariant targets appear more different than their backgrounds in lightness than do texture-variant targets. Overall, the white line patterns yielded slightly darker matches for the targets. Like the previous experiments in this study, postexperimental inquiries indicated that the texture-variant targets presented in Experiment 3 appeared as 3-D entities that were not coplanar with their backgrounds.

\section{GENERAL DISCUSSION}

The results of the present study suggest that under our experimental conditions, when targets and backgrounds shared a common line-texture pattern, perceived contrast could be obtained. When targets are superimposed with a line pattern that is different than the pattern superimposed onto the target's background, contrast is significantly reduced. Clearly there was some characteristic in the line patterns that provided the visual system with information that can affect both lightness contrast and inferred depth relationships. That characteristic might have been T-junctions.

These results generally agree with those obtained by Laurinen et al. (1997), but the results of the present study suggest conclusions that go beyond their findings. In Experiments 2 and 3, the targets were presented on the same background.This disambiguated the effects of modulation patterns and differential effects of retinal lateral inhibition for light and dark backgrounds, because lateral inhibition was equated for both targets. Versions of the classic SLC display have been used extensively in lightness research, but for the variables addressed in the present study, a common background for both targets has value, in that the effects of the texture patterns were isolated.

The results of the present study can be discussed in terms of both Anderson's (1997) scission theory and Gilchrist et al.'s (1999) anchoring theory of lightness. In Anderson's model, the visual system sometimes separates a given region of luminance into two layers (scission): (1) a background layer, and (2) a translucent filter layer, or illumination layer, that lies on top of the background layer. For the present, Anderson's model is perhaps best illustrated when applied to White's (1981) effect (see Figure 2). The corners of the gray targets form T-junctions because three distinct luminance regions come together at that point. The stem of the $\mathrm{T}$ is formed by the contour between the gray target and the stripe on which it appears to be continuous. This contour is treated by the visual system as either the edge of a transparent layer or as a change in illumination. To be concrete, some of the darkness of the gray targets that appear to be on black stripes is attributed to the black background beneath, making the targets appear lighter. Conversely, some of the lightness of the gray targets that appear to be on white stripes is attributed to the white background layer beneath, making the targets appear darker. Anderson's model can be applied to the texture-invariant targets in the present study. Texture-invariant targets and background regions constitute the stems of many T-junctions in those displays (see Figure 8b). Similar to White's effect, the texture-invariant targets on darker backgrounds might have appeared lighter than their texture-variant counterparts because some of their darkness was attributed to the dark background layers. The same reasoning can be applied to the targets on light backgrounds. Because the regions on either side of the stems in the texture-invariant displays were not composed of target and background regions, no scission of the target region could occur.

According to the anchoring theory of lightness perception (Gilchrist et al., 1999), the highest luminance within a perceptual framework is locally assigned the role of white, and lesser luminance values are perceived relative to this anchor. For example, in an SLC display, one can think of two local frameworks side by side. The gray target on the black background is locally assigned the role of white because it has the highest luminance in that local framework. The target on the white background is not the anchor in that local framework, the white background itself having fulfilled that role. However, the gray target on the black background does not appear white because the larger context of a scene (the global framework) sometimes contains regions that have a luminance value even higher than that of the target on black (in this case, the white background). As a result, the lightness of the gray target on black is based on a compromise between the anchors in the local and global frameworks. Because the global framework is larger and more complex, the influence of its anchor on the target surrounded by black is much stronger than the influence of the local anchor (the target itself). However, the local anchor still exerts some influence on the target, making it appear slightly lighter than the target surrounded by white.

Consider the increment display shown in Figure 5. An analysis of this display in terms of anchoring is as follows: The observers reported that the texture-invariant target on the left appeared to be behind the lines of the background, whereas the texture-variant target appeared as a separate 3-D entity positioned some distance in front of the display. The texture-invariant target, which appears to be behind the lines of the background, becomes a member of a local framework that also includes the dark gray background. This target will tend to move toward white because it is the highest luminance within its local frame. The texture-variant target, which appears to be positioned on top of the background lines, is more influenced by the global framework, which has its own anchor- 
namely, a real white surface whose luminance is higher than that of the target.

For the decrement display, the texture-invariant target also appears to be behind the lines of the background, and thus it also constitutes a local framework that includes the white background. However, this target will not move towards white because it does not have the highest luminance within its local framework; in this display, it is the white background itself that plays the role of local anchor. Like the increment display, the texture-variant target is most heavily influenced by the global framework. In short, the anchoring model of lightness perception would predict a larger lightness difference for the targets of the increment display. Any differences for the targets for the decrement displays would be the effect of the scale-normalization component of the model-a component that has been shown to be much weaker than the anchoring component. In the present study, significant differences were found for both increment and decrement displays; however, the results cannot be used to determine whether the target differences for the increment displays were larger than the differences for the decrement displays.

In the present study, we addressed the question of whether increments and/or decrements are affected by texture patterns. Increment and decrement displays were tested separately, and significant lightness differences for the targets were found for both displays. In SLC displays, some investigators (e.g., Diamond, 1953, 1955; Heinemann, 1955; Leibowitz, Mote, \& Thurlow, 1953) have proposed that the gray target surrounded by white (decrement target) is affected more by lateral inhibition than is the target surrounded by black (increment target). When this idea is applied to the results of the present study, the differential effects of lateral inhibition on increments and decrements can explain little, given the fact that in Experiments 2 and 3, the targets on each display were both either increments or decrements. Thus inhibition exerted on the targets by surrounding regions would have been the same for any pair of targets in Experiments 2 and 3 .

The effects of black and white line-texture patterns were tested separately in order to determine whether line-texture patterns affect perceived lightness contrast via lightness assimilation. Black and white line patterns yielded similar results. In general, targets superimposed with light lines appeared darker than targets superimposed with dark lines, suggesting a contrast effect-not assimilation. However, targets that shared a line-texture pattern with their respective backgrounds appeared more different from that background in terms of lightness than did targets that did not share a texture pattern.

Collectively, the results of the present study support an explanation based on T-junctions. The idea that T-junctions can affect lightness and lightness contrast is not a new one, and explanations based on factors other than depth and occlusion are plausible. Others (e.g., Anderson, 1997; Gilchrist et al., 1999; Todorović, 1997) have made the claim, differing on the specifics of how T-junctions work, but in general agreeing, that T-junctions can affect perceptual grouping, and in turn, perceived contrast. However, because differences in lightness judgments in the present study were accompanied by differences in perceived depth, it is also plausible that T-junctions affect lightness via the information they provide about occlusion. Although more work is clearly needed to more fully understand how T-junctions affect lightness, the perceived depth differences they produce may be crucial.

Consider the T-junction shown in Figure 8a. The two regions on either side of the T-junction's stem (A and B) are generally perceived to be coplanar. The region whose border is perpendicular to the stem of the $\mathrm{T}$ (Region C) is generally perceived to be on a different depth plane. It seems to be a general finding that regions that are

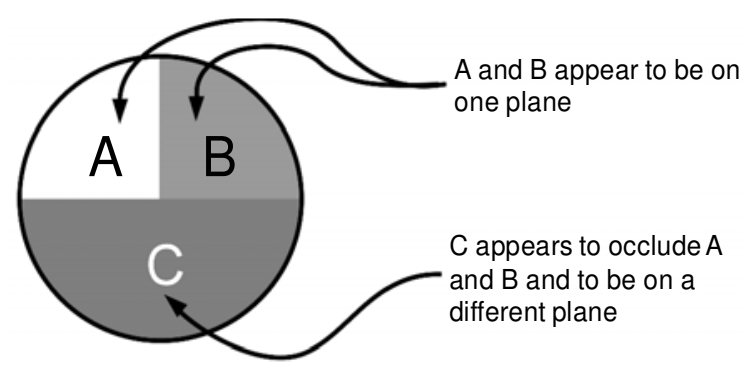

a) T-junction

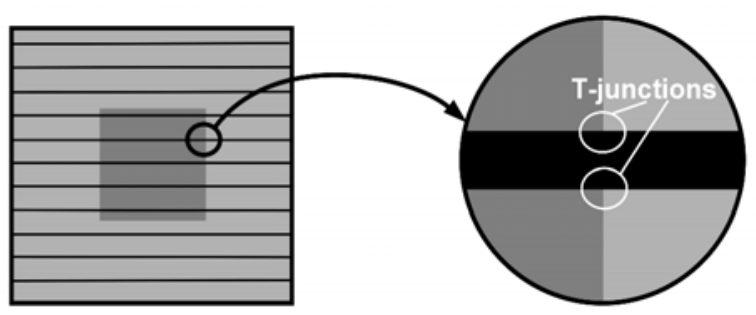

b) Texture-invariant T-junction

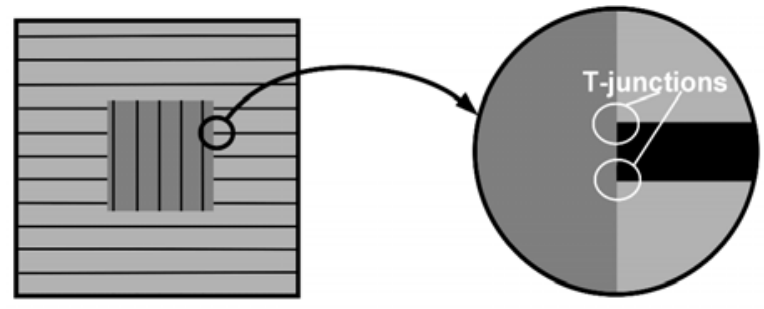

c) Texture-variant T-junction

Figure 8. (a) Regions A and B are perceived to be on one plane. Region $C$ is perceived to occlude Regions $A$ and $B$ and is perceived to be on a separate plane. (b) T-junctions at the border of the texture-invariant target provide information leading to a perceived "screen" positioned in front of the background and texture-invariant target. (c) T-junctions at the borders of the texture-variant target provide information that indicates that the texture-variant target lies on a separate depth plane than does the background. 


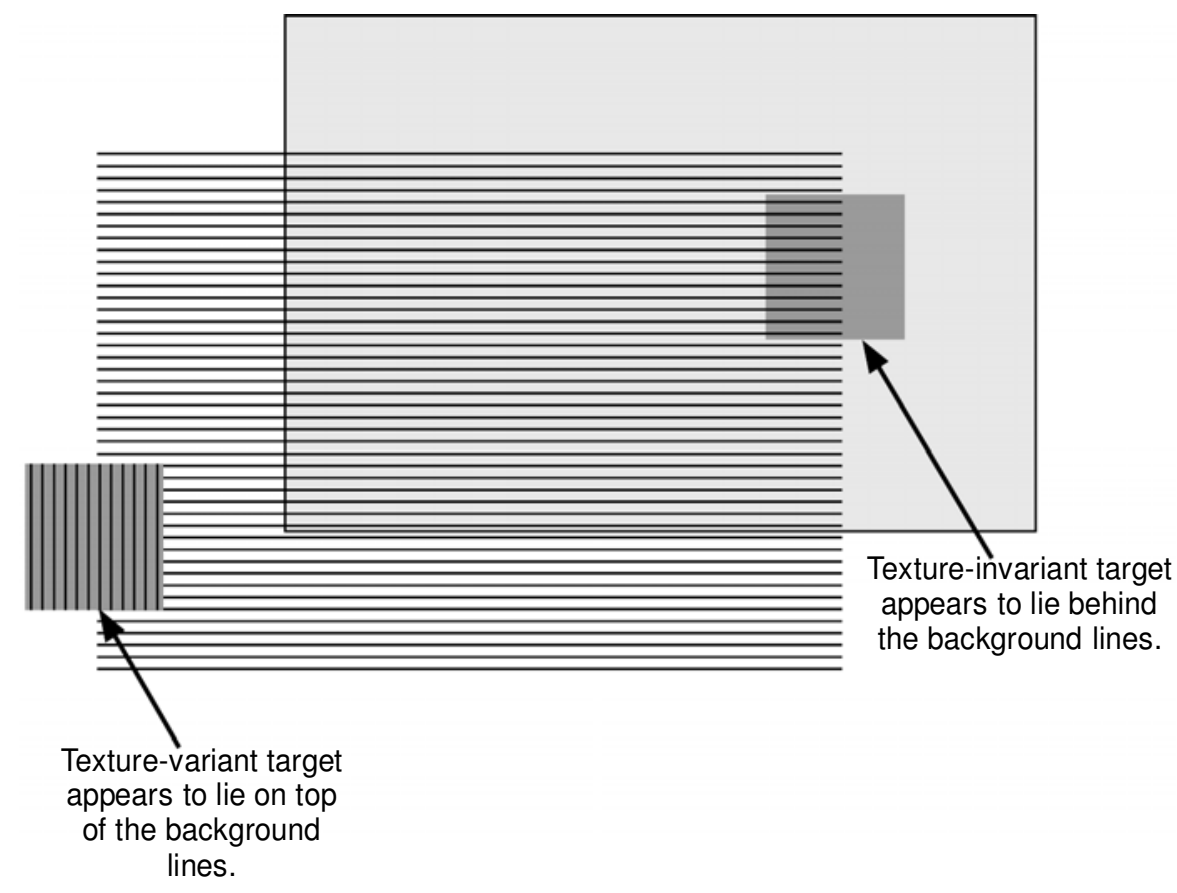

Figure 9. Layer percept of line-texture displays used in the present study. The texture-invariant target and the background form one layer, the lines superimposed onto the background form another layer, and the texture-variant target appears as a third layer in front of the other two.

grouped together are compared more with each other and lightness contrast can be the outcome. This type of analysis might account for the Benary Cross (Benary, 1924) and the enigmatic White's effect (White, 1981), in which a strong contrast effect is evident, but one that goes in the opposite direction of what a lateral inhibition theory would predict.

T-junctions could have affected the apparent or registered depth of both the texture-invariant and texture-variant targets presented in Experiments 1-3 but in different ways. T-junctions formed at the borders of the texture-invariant targets cause the common line pattern shared by target and background to appear as a "screen" or "fence" that is located on a separate plane. The line pattern is seen as a separate layer positioned between the observer and the display's background. An illustration of this layered type of percept is shown in Figure 9. The texture-invariant target and its background appear to be more or less coplanar. The texture-variant target appears to pop out in depth, hovering some distance in front of the rest of the display, including the screen. In all, at least three distinct layers are perceived: the background and texture-invariant target, the "screen" that is composed of the lines on the background, and the texture-variant target that appears to float in front of the first two layers.

T-junctions at the borders of the texture-invariant and texture-variant targets provide information about where the targets appear relative to the screen layer. Figure $8 \mathrm{~b}$ shows how T-junctions formed at the border of the textureinvariant targets produce the impression of an occluding screen or fence positioned in front of the texture-invariant target and the background. Figure $8 \mathrm{c}$ shows how T-junctions formed at the border of the texture-variant target produce the impression of the texture-variant target occluding the screen and the background.

The idea that surfaces that appear to be closer to one another in 3-D space are compared more with one another is also not a new idea. In his study of lightness constancy, Gilchrist $(1977,1980)$ showed that surfaces perceived to be coplanar are typically compared with one another, and surfaces perceived to be on different planes are not. Gilchrist's coplanar ratio rule was meant to apply to scenes in which the overall luminance range exceeds 30:1. Although the luminance range of the displays in the present study did exceed $30: 1$, grouping by coplanarity could still have played a role. Using computer-generated displays and stereoscopic presentations, Schirillo, Reeves, and Arend (1990) obtained results similar to those obtained by Gilchrist. Gogel and Mershon (1969) showed that the more adjacent surfaces appear to be, the more they are compared with one another in terms of their lightness. The idea of grouping because of spatial arrangement perhaps goes back as far as the early Gestaltists (Wertheimer, 1923), who showed that regions close to one another in terms of spatial proximity tend to form a perceptual group. The line patterns used in Experiments 1-3 
affected inferred depth, and the net result was that the texture-invariant targets were grouped and compared more with their respective backgrounds.

\section{REFERENCES}

Adelson, E. H. (1993). Perceptual organization and the judgment of brightness. Science, 262, 2042-2044.

Agostini, T., \& Proffitt, D. R. (1993). Perceptual organization evokes simultaneous lightness contrast. Perception, 22, 263-272.

Anderson, B. (1997). A theory of illusory lightness and transparency in monocular and binocular images: The role of contour junctions. Perception, 26, 419-453.

Benary, W. (1924). Beobachtungen zu einem Experiment über Helligkeitskontrast [Observations from an experiment on brightness contrast]. Psychologische Forschung, 5, 131-142.

CoRen, S. (1969). Brightness contrast as a function of figure-ground relations. Journal of Experimental Psychology, 80, 517-524.

Cornsweet, T. N. (1970). Visual perception. New York: Academic Press.

Diamond, A. L. (1953). Foveal simultaneous contrast as a function of inducing and test-field luminance. Journal of Experimental Psychology, 45, 304-314.

Diamond, A. L. (1955). Foveal simultaneous contrast as a function of inducing-field area. Journal of Experimental Psychology, 50, 144-152.

Diamond, A. L. (1960). A theory of depression and enhancement in the brightness response. Psychological Review, 67, 168-199.

Economou, E., Annan, V., \& Gilchrist, A. L. (1998). Contrast depends on anchoring in perceptual groups [Abstract]. Investigative Ophthalmology \& Visual Science, 39, S857.

GILCHRIST, A. L. (1977). Perceived lightness depends on perceived spatial arrangement. Science, 195, 185-187.

GILCHRIST, A. L. (1980). When does perceived lightness depend on perceived spatial arrangement? Perception \& Psychophysics, 28, 527-538.

GILCHRIST, A. L. (1988). Lightness contrast and failures of constancy: A common explanation. Perception \& Psychophysics, 43, 415-424.

Gilchrist, A. L., Kossy fidis, C., Bonato, F., Agostini, T., Cataliotti, J., Li, X., Spehar, B., Annan, V., \& Economou, E. (1999). An anchoring theory of lightness perception. Psychological Review, 106, $795-834$.
Gogel, W. C., \& Mershon, D. H. (1969). Depth adjacency in simultaneous contrast. Perception \& Psychophysics, 5, 13-17.

Hartline, H. K., Wagner, H. G., \& Ratliff, F. (1956). Inhibition in the eye of Limulus. Journal of General Physiology, 39, 651-673.

HeInEMANN, E. G. (1955). Simultaneous brightness induction as a function of inducing and test-field luminances. Journal of Experimental Psychology, 50, 89-96.

JAmeson, D., \& Hurvich, L. M. (1961). Complexities of perceived brightness. Science, 133, 174-179.

JAmeson, D., \& Hurvich, L. M. (1964). Theory of lightness and color contrast in human vision. Vision Research, 4, 135-154.

Laurinen, P. I., Olzak, L. A., \& Peromaa, T. L. (1997). Early cortical influences in object segregation and the perception of surface lightness. Psychological Science, 8, 386-390.

Leibowitz, H., Mote, F. A., \& Thurlow, W. R. (1953). Simultaneous contrast as a function of separation between test and inducing fields. Journal of Experimental Psychology, 46, 453-456.

Nabet, B., \& Pinter, R.B. (1991). Sensory neural networks: Lateral inhibition. Boca Raton, FL: CRC Press.

RATLIFF, F. (1965). Mach bands: Quantitative studies on neural networks in the retina. New York: Holden-Day.

Schirillo, J., Reeves, A., \& Arend, L. (1990). Perceived lightness, but not brightness, of achromatic surfaces depends on perceived depth information. Perception \& Psychophysics, 48, 82-90.

Shapley, R, Caelli, T., Grossberg, S., Morgan, M., \& Rentschler, I. (1990). Computational theories of visual perception. In L. Spillman \& J. Werner (Eds.), Visual perception: The neurophysiologicalfoundations (pp. 231-272). New York: Academic Press.

Todorović, D. (1997). Lightness and junctions. Perception, 26, 379-394.

WerTheimer, M. (1923). Untersuchungen zur Lehre von der Gestalt: II [Investigations in Gestalt theory: II]. Psychologische Forschung, 4, 301-350.

White, M. (1981). The effect of the nature of the surround on the perceived lightness of grey bars within square-wave test gratings. Perception, 10, 1493-1507.

WoLfF, W. (1933). Concerning the contrast-causing effect of transformed colors. Psychologische Forschung, 18, 90-97.

(Manuscript received November 9, 1999; revision accepted for publication February 19, 2002.) 